\title{
Ophthalmologic Complication
}

National Cancer Institute

\section{Source}

National Cancer Institute. Ophthalmologic Complication. NCI Thesaurus. Code C115319.

Any eye disorder occurring as a consequence of injury to the eye. 